\title{
Effect of Graston Technique on Fatigue Recovery after High-Intensity Exercise of the Quadriceps Femoris
}

\author{
Ho-Hee Son ${ }^{\dagger}$ \\ Department of Physical Therapy, College of Health Sciences, Catholic University of Pusan
}

Received: July 4, 2018 / Revised: July 16, 2018 / Accepted: August 9, 2018

(C) 2018 J Korean Soc Phys Med

\section{| Abstract |}

PURPOSE: This study was conducted to compare the effects of the Graston-based massage method and microcurrent application on muscle fatigue after causing muscle fatigue of the quadriceps femoris by applying highintensity exercise.

METHODS: Study subjects (56 healthy subjects in their 20s) were randomized into a microcurrent group (MG), Graston group (GG), and control group (CG). To induce fatigue of the quadriceps femoris, the subjects performed squats 100 times without a break within about 5 minutes. Muscle fatigue was measured immediately after performing squats and 15 minutes after intervention. To measure muscle fatigue, surface electromyogram (EMG) was used to obtain and compare the median frequency. Microcurrent or Graston massage was applied to their quadriceps muscles 15 minutes after performing the squats.

RESULTS: Muscle fatigue increased significantly in the

†Corresponding Author : Ho-Hee Son sonhh@cup.ac.kr, http://orcid.org/0000-0003-0905-6484

This is an Open Access article distributed under the terms of the Creative Commons Attribution Non-Commercial License (http://creativecommons.org/licenses/by-nc/3.0) which permits unrestricted non-commercial use, distribution, and reproduction in any medium, provided the original work is properly cited. vastus lateralis before and after intervention in the Graston group $(p<.05)$, while no significant difference was observed in the rectus femoris and vastus medialis ( $>>05)$. There were also no significant differences in the microcurrent group or the control group.

CONCLUSION: Both the microcurrent group and Graston group showed a tendency for increasing median frequency values compared to the control group, but there was no significant difference except in the vastus lateralis treated with the Graston technique. Although there was no significant difference, the Graston technique could be utilized to reduce the occurrence of DOMS by preventing muscle fatigue in clinical practice or sports medicine.

Key Words: Graston massage, Microcurrent, Muscle fatigue

\section{Introduction}

With the recent increased interest in health, various exercises have been proposed to improve physical strength. To maintain health, modern people are performing high-intensity exercises, such as climbing, marathons, and squats to maximize the effects of exercise in the short term. Exercising at appropriate intensity can slow down aging and induce immunosuppression, resulting in reduced risk of infectious diseases (Nieman et al., 1994; Gomes et al., 2018). However, high-intensity exercise or intense physical 
activities can induce oxidative stress via the accumulation of fatigue from increased lactic acid and increased active oxygen (Joo, 2008; Senturk et al., 2005). Thus, various methods, such as cooling down or stretching after exercising (Yokoe and Ohira, 2017), cold therapy (Eguchi et al., 2014; Kang et al., 2018), electrotherapy (Lou et al., 2017), and massage (Mori et al., 2004) are suggested to relieve muscle fatigue after high-intensity exercise. Among them, electrotherapy for reducing muscle fatigue to treat delayed onset muscle soreness (DOMS) includes electric stimulation therapy, transcutaneous electrical nerve stimulation, and microcurrent therapy. Unlike low-frequency therapy and transcutaneous electrical nerve stimulation, which stimulate at the $\mu \mathrm{A}$ level, microcurrent increases the voltage to reduce the current, as occurs during infection and tissue renewal (Todd et al., 2001). Various studies have shown that microcurrent treatment is effective at improving muscle function, soft tissue healing, fracture healing, and angiogenesis (Kwon et al., 2017; Fujiya et al., 2015; Whitcomb et al., 2012).

Massage, which is also used to relieve muscle fatigue, has been reported to be effective in fatigue recovery by promoting circulation of the capillary vessels and relaxing muscle tension (Hemmings et al., 2000; Moraska, 2007). Recovery of muscle fatigue can be increased by combining massage with rest after exercise (Mori et al., 2004). In clinical settings, tools such as the Graston technique are used to make massage easier than using hands alone. Myofascial release using the Graston technique is known to reduce pain, reorganize tissues, and ultimately promote functional movement, and is commonly used in clinical practice as a method of instrument assisted soft tissue mobilization (IASM) (Stanek et al., 2018). Gehlsen et al. (1999) reported that the controlled microtrauma induced using the Graston technique increased fibroblasts recruitment and activation in an animal model. Recently, various studies utilizing the Graston, such as applying it to increase range of motion (ROM) in order to increase flexibility of the quadriceps femoris and reduce back pain (Kim et al., 2014; Moon et al., 2017) or applying it to subacute lumbar compartment syndrome patients for muscle relaxation (Hammer and Pfefer, 2005), have been conducted. However, studies of the effects of Graston-based massage on muscle fatigue have been rare.

As mentioned previously, microcurrent or massage are commonly used to relieve muscle fatigue after exercise. However, no studies have directly compared the immediate effect of Graston-based massage and microcurrent on muscle fatigue after high-intensity exercise.

Therefore, this study was conducted to compare the effects of the Graston-based massage method and microcurrent application on muscle fatigue after causing muscle fatigue in the quadriceps femoris by applying high-intensity exercise.

\section{Methods}

\section{Subject}

This study investigated 60 healthy subjects in their $20 \mathrm{~s}$. After four dropouts, a total of 56 adults were included in the analysis. The inclusion criteria were 1) physically healthy adults in their 20s, 2) individuals without a medical history of musculoskeletal disease in the past 2 years or experience of pain, and 3) individuals who were able to perform squats. Exclusion criteria included 1) a BMI of more than 23 and 2) individuals who regularly performed high-intensity exercise more than 2 times a week.

Study subjects were randomized into the microcurrent group (MG), Graston group (GG), and control group (CG). In addition, the subjects were informed about the purpose, process, and significance of the study prior to beginning the study and voluntarily agreed to participate.

\section{Measurement method and tool}

To induce fatigue of the quadriceps femoris, squat exercises were used. When performing squats, the subjects 
had their feet positioned at shoulder width with their arms crossed in front of their chest while maintaining the trunk straight. The subjects performed squats 100 times without a break within about 5 minutes (Ahn et al., 2015). Four subjects were excluded due to failure to perform the squat exercise. Measurement of muscle fatigue was conducted immediately after performing squats and 15 minutes after intervention

To measure muscle fatigue, a surface electromyogram (EMG) (WEMG8, Laxtha, Korea) was used to obtain and compare the median frequency (Hong et al., 2003). In addition, the telescan program was used to analyze the raw data. The sampling frequency was set to $1024 \mathrm{~Hz}$, which was calculated based on the square root of the RMS value after squaring and integration. To remove the noise associated with the electronic equipment, band-pass filtering was performed at 10-500 $\mathrm{Hz}$. For the EMG signal, 20 seconds of data obtained by excluding the first and last 5 seconds of a 30 second signal recorded before and after the intervention were processed with FFT.

The distance between the centers of the electrodes was $2 \mathrm{~cm}$. To prevent measurement errors, the electrodes were cleaned with medical alcohol after shaving, then attached to the vastus lateralis, rectus femoris and vastus medialis. For the vastus lateralis, the electrodes were attached to the lateral side of the femur at $3-5 \mathrm{~cm}$ from the midline, and the electrodes for the rectus femoris were attached halfway between the knee and iliac spine. The electrodes for the vastus medialis were attached to the point two-thirds from the knee to the iliac spine (Cram et al., 1990).

\section{Intervention method}

\section{1) Microcurrent application}

An EMDOMED682 (Enraf-Nonius, Netherlands) microcurrent device was used with two electrodes. One electrode was attached to the distal part of the vastus lateralis and the other was attached to the distal part of the vastus medialis. The frequency of the current was set at $30 \mathrm{pps}$ with an intensity of $100 \mu \mathrm{A}$. Current was applied 15 minutes after performing squats (Kim et al., 2009).

\section{2) Graston application}

For Graston-based massage, a GT-1 (FROG, Frogfitness, China) system was used. For the massage, the subject lied down and massage cream was applied to the quadriceps femoris. The convex part of the Graston was then applied to each muscle in the direction from cranial to caudal for 2 minutes following the order of vastus lateralis, rectus femoris and vastus medialis.

\section{3) Control group}

For the control group, subjects rested in a sitting position for 15 minutes in the same space immediately after performing the squats.

\section{Statistical analysis}

For data analysis, IBM SPSS ver 24.0 for windows was used. After confirming the normality of the general characteristics of the microcurrent group, Graston group and control group, as well as the muscle fatigue test before intervention, one-way ANOVA was conducted. In addition, paired t-tests were performed to identify significant differences in muscle fatigue before and after intervention in each group. A $p<.05$ was considered to indicate statistical significance.

\section{Results}

\section{General characteristics of subjects}

The demographic characteristics of the 56 participants are shown in Table 1 . There were no significant differences in variables.

\section{Median frequency of each muscle in three groups before intervention \\ Immediately after high-intensity exercise, the median}


Table 1. General Characteristics of Subjects

\begin{tabular}{lccc}
\hline \multicolumn{1}{c}{ Variables } & MG $(\mathrm{n}=18)$ & GG $(\mathrm{n}=20)$ & CG $(\mathrm{n}=18)$ \\
\hline Sex (male/female) & $10 / 8$ & $10 / 10$ & $10 / 8$ \\
Age (yrs) & $22.61 \pm .92$ & $22.15 \pm 1.31$ & $21.45 \pm 1.68$ \\
Height (cm) & $163.57 \pm 9.91$ & $165.53 \pm 7.92$ & $164.94 \pm 7.50$ \\
Weight $(\mathrm{kg})$ & $57.37 \pm 12.32$ & $61.32 \pm 11.04$ & $59.57 \pm 6.55$ \\
\hline
\end{tabular}

MG ; microcurrent group, GG ; Graston massage group, CG ; control group

Table 2. Median Frequency of Each Muscle before Intervention

\begin{tabular}{cccccc}
\hline Variables $(\mathrm{Hz})$ & MG $(\mathrm{n}=18)$ & GG $(\mathrm{n}=20)$ & CG $(\mathrm{n}=18)$ & $\mathrm{F}$ & $\mathrm{p}$ \\
\hline Vastus Lateralis & $48.41 \pm 6.11$ & $54.88 \pm 12.36$ & $53.67 \pm 9.75$ & 2.21 & .12 \\
Rectus Femoris & $70.38 \pm 16.83$ & $82.31 \pm 19.84$ & $85.85 \pm 15.55$ & 1.92 & .11 \\
Vastus Medialis & $46.40 \pm 4.57$ & $50.37 \pm 4.04$ & $56.08 \pm 15.67$ & 3.07 & .06 \\
\hline
\end{tabular}

MG ; microcurrent group, GG ; Graston massage group, $\mathrm{CG}$; control group

Table 3. Median Frequency of Microurrent Group before and after Intervention $(n=18)$

\begin{tabular}{cllll}
\hline MG $(\mathrm{Hz})$ & \multicolumn{1}{c}{ pre } & post & $\mathrm{t}$ & $\mathrm{p}$ \\
\hline Vastus Lateralis & $48.41 \pm 6.11$ & $48.43 \pm 7.13$ & -.12 & .99 \\
Rectus Femoris & $70.38 \pm 16.83$ & $77.54 \pm 23.53$ & -1.89 & .08 \\
Vastus Medialis & $46.40 \pm 4.57$ & $46.36 \pm 4.51$ & -.43 & .97 \\
\hline
\end{tabular}

$\mathrm{MG}$; microcurrent group

frequencies of the vastus lateralis, rectus femoris and vastus medialis were measured. No significant differences were observed among groups (Table 2).

3. Median frequency of microcurrent group before and after intervention

There were no significant differences in any muscles in the microcurrent group (Table 3).

\section{Median frequency of Graston group before} and after intervention

The muscle fatigue in the Graston group increased significantly in the vastus lateralis $(p<.05)$, while no significant difference was observed in the rectus femoris or vastus medialis ( $\mathrm{p}>.05)$ (Table 4).
5. Median frequency of control group before and after intervention

No significant differences were observed in any of the muscles in the control group (Table 5).

\section{Discussion}

This study was conducted to identify the effects of microcurrent and Graston-based massage on muscle fatigue caused by high-intensity exercise. To cause muscle fatigue, high-intensity squat exercise was performed. The results showed that muscle fatigue of the vastus lateralis after squat exercise was significantly reduced in the Graston group, while there was no significant difference in any muscles before and after exercise in the microcurrent and Control groups. 
Table 4. Median Frequency of Graston Massage Group before and after Intervention $(n=20)$

\begin{tabular}{ccccc}
\hline GG $(\mathrm{Hz})$ & pre & post & $\mathrm{t}$ & $\mathrm{p}$ \\
\hline Vastus Lateralis & $54.88 \pm 12.36$ & $60.36 \pm 14.72$ & -2.56 & .02 \\
Rectus Femoris & $82.31 \pm 19.84$ & $82.84 \pm 17.59$ & -.21 & .83 \\
Vastus Medialis & $50.37 \pm 4.04$ & $54.51 \pm 11.16$ & -1.59 & .13 \\
\hline
\end{tabular}

GG; Graston massage group

Table 5. Median Frequency of Control Group before and after Intervention $(n=18)$

\begin{tabular}{ccccc}
\hline CG $(\mathrm{Hz})$ & Pre & post & $\mathrm{t}$ & $\mathrm{p}$ \\
\hline Vastus Lateralis & $53.67 \pm 9.75$ & $54.01 \pm 10.03$ & -.27 & .79 \\
Rectus Femoris & $85.85 \pm 15.55$ & $82.49 \pm 15.29$ & 1.06 & .30 \\
Vastus Medialis & $56.08 \pm 15.67$ & $56.97 \pm 17.33$ & -.23 & .82 \\
\hline
\end{tabular}

CG; control group

Muscle fatigue, which is defined as reduced maximum muscle strength that a muscle can exert, can develop because of repeated activity of the muscle and leads to reduced ability to produce strength (Millet and Lepers, 2004). The incidence of muscle fatigue varies depending on the duration and intensity of exercise, but it can be a risk factor that causes pain or musculoskeletal disorders. Surface EMG, which is a non-invasive method, can be used to objectively measure fatigue in muscles (Elfving et al., 2002). The median frequency analyzed based on the effective value amplitude, which reflects the amplitude of EMG frequency and the frequency spectrum, as well as the mean frequency, are considered reliable indices of fatigue indexes (Jurell, 1998; De Luca, 1984; Kienbacher et al., 2014). When muscle fatigue occurs, increases in electric power at low frequency and decreases of electric power at high frequency or decreases of the low frequency to high frequency ratio (Badier et al., 1993) cause the median frequency of the muscle to shift to a lower frequency range (Lyons et al., 1993; Stulen and De Luca, 1982).

In this study, there were no significant differences in muscle fatigue after microcurrent therapy. Microcurrent therapy is a type of subsensory stimulation applied as an intervention method mainly for pain, edema and inflammation (Craig et al., 1996). Curtis et al. (2010) investigated the effects of microcurrent on DOMS by conducting high-intensity eccentric exercise in subjects and applied microcurrent for 20 minutes immediately after exercise. They found that pain intensity was significantly decreased compared to the control group, even after 72 hours.

Lambert et al. (2002) reported that microcurrent treatment reduced the signs and symptoms of muscle damage after an eccentric exercise protocol and that this was likely related to maintenance of intracellular $\mathrm{Ca}^{2+}$ homeostasis after muscle damaging exercise. Lee et al. (2013) also applied microcurrent for 4 days to treat DOMS that developed after muscle fatigue. They found that the $\mathrm{R}$ reflex measured by EMG was significantly reduced as time passed. Because this study was conducted to identify the immediate effects of microcurrent, changes according to time were not observed. Although not significant immediately after applying microcurrent, the median frequency of the rectus femoris in the control group decreased, while that in the microcurrent group increased, showing a tendency for decrease in muscle fatigue.

Massage is widely used in sports medicine or clinical practice as it is considered effective at the recovery of muscle fatigue (Hemmings et al., 2000). Tanaka et al. 
(2002) compared a control group and Graston group that underwent 5 minutes of massage after inducing muscle fatigue in the back region. Measurements of median frequency using EMG showed a significant decrease in VAS in the group that received massage, but there was no significant difference in muscle fatigue.

Recently, tools have been used to conduct massages or IASM in clinical practice, but there is not much evidence of their effectiveness. Among many methods of massage, the Graston technique is used to treat mechanical micro-traumatic damage by IASM, and it has been reported to effectively promote adhesion of the treated tissues and collagen synthesis (Gehlsen et al., 1999). In an RCT study of manipulative therapy conducted by Crothers et al. (2016), application of the Graston technique to patients with pain in thoracic vertebrae resulted in no significant effects, but all subjects reported that all pain indexes decreased with time. Lee et al. (2016) also reported a significant increase in pain and ROM after applying the Graston technique to chronic backache patients. However, few studies have investigated application of the Graston technique for recovery of muscle fatigue, and no studies have investigated the immediate effects of this treatment using EMG signals.

Moon et al. (2017) reported an immediate effect of Graston-based massage on pain and hamstring extensibility. However, Ce et al. (2013) found no change in the blood acidic acid concentration when stretching and massages were performed for 10 minutes after high-intensity exercise. Resnick (2016) also found no metabolic changes in cell level when massage was performed for 30 minutes after exercise, and there were no significant differences in EMG signal. In this study, all median frequency values of the EMG signal increased immediately after applying the Graston technique, but significant differences were only found in the vastus lateralis among the three muscles. About 6 minutes of Graston-based massage stimulation was applied, but this seems to have been insufficient to induce significant changes in the EMG signal.
In this study, Graston-based massage and microcurrent were applied after performing high-intensity exercise to assess muscle fatigue through changes in median frequency using surface EMG. The purpose was to investigate the immediate effect after intervention. The two groups that received the Graston technique and microcurrent showed a tendency for increasing median frequency values compared to the control group, but there were no significant differences except for in the vastus lateralis treated by the Graston technique.

It should be noted that this study is limited in that although microcurrent and the Graston technique were applied after causing muscle fatigue to observe their immediate effects, the effects on DOMS were not observed through follow up. In addition, because the analysis of muscle fatigue was based only on the median frequency, which is one of the EMG signals, the effects on pain were not observed. Therefore, further study is needed to investigate the effects of the Graston technique on muscle fatigue based on time and various measurements.

\section{Conclusion}

This study investigated the immediate effects of applying Graston-based massage and microcurrent on muscle fatigue after high-intensity exercise. Although there were no significant differences observed, the results indicated that the method could be utilized to prevent muscle fatigue in clinical practice or sports medicine.

\section{Acknowledgements}

This paper was supported by a RESEARCH FUND from Catholic University of Pusan and the National Research Foundation of Korea (NRF) funded by the Korea government (MSIT) No. 2017R1C1B5074040. 


\section{References}

Ahn I, Jeon J, Kim D. The Immediate Effects of Kinesio Taping on Quadriceps Induced Fatigue by Short-term Squat. The Journal of Korean Academy of Orthopecic Manual Therapy. 2015;21(1):21-8.

Badier M, Guillot C, Lagier-Tessonnier F, et al. EMG power spectrum of respiratory and skeletal muscles during static contraction in healthy man. Muscle Nerve. 1993;16(6):601-9.

Ce E, Limonta E, Maggioni MA, et al. Stretching and deep and superficial massage do not influence blood lactate levels after heavy-intensity cycle exercise. J Sports Sci. 2013;31(8):856-66.

Craig JA, Barlas P, Baxter GD, et al. Delayed-onset muscle soreness: lack of effect of combined phototherapy/ low-intensity laser therapy at low pulse repetition rates. J Clin Laser Med Surg. 1996;14(6):375-80.

Cram JR, Lloyd J, Cahn TS. The reliability of EMG muscle scanning. Int J Psychosom. 1990;37(1-4):68-72.

Crothers AL, French SD, Hebert JJ, et al. Spinal manipulative therapy, Graston technique(R) and placebo for non-specific thoracic spine pain: a randomised controlled trial. Chiropr Man Therap. 2016;24:16.

Curtis D, Fallows S, Morris M, et al. The efficacy of frequency specific microcurrent therapy on delayed onset muscle soreness. J Bodyw Mov Ther. 2010;14(3):272-9.

De Luca CJ. Myoelectrical manifestations of localized muscular fatigue in humans. Crit Rev Biomed Eng. 1984; 11(4):251-79.

Eguchi $\mathrm{Y}$, Jinde M, Murooka $\mathrm{K}$, et al. Stretching versus transitory icing: which is the more effective treatment for attenuating muscle fatigue after repeated manual labor? Eur J Appl Physiol. 2014;114(12):2617-23.

Elfving B, Liljequist D, Dedering A, et al. Recovery of electromyograph median frequency after lumbar muscle fatigue analysed using an exponential time dependence model. Eur J Appl Physiol. 2002;88(1-2):
$85-93$.

Fujiya H, Ogura Y, Ohno Y, et al. Microcurrent electrical neuromuscular stimulation facilitates regeneration of injured skeletal muscle in mice. J Sports Sci Med. 2015;14(2):297-303.

Gehlsen GM, Ganion LR, Helfst R. Fibroblast responses to variation in soft tissue mobilization pressure. Med Sci Sports Exerc. 1999;31(4):531-5.

Gomes Neto M, Duraes AR, Conceicao LSR, et al. High intensity interval training versus moderate intensity continuous training on exercise capacity and quality of life in patients with heart failure with reduced ejection fraction: A systematic review and meta-analysis. Int J Cardiol. 2018;261:134-41.

Hammer WI, Pfefer MT. Treatment of a case of subacute lumbar compartment syndrome using the Graston technique. J Manipulative PhysiolTher. 2005;28(3): 199-204.

Hemmings B, Smith M, Graydon J, et al. Effects of massage on physiological restoration, perceived recovery, and repeated sports performance. Br J Sports Med. 2000;34(2):109-14.

Hong SY, Lee SH, Yoon HR, et al. The Study of Muscle Fatique Index Searching in terms of Median Frequency Analysis of EMG Signals during Isotonic Exercise. Journal of Biomedical Engineering Research. 2003;24(3):175-81.

Joo M. Changes of Blood Oxidative Stress Markers after a High-Intensity Exercise in Young Women. Korean journal of health promotion and disease prevention. 2008;8(3):150-7.

Jurell KC. Surface EMG and fatigue. Phys Med Rehabil Clin N Am. 1998;9(4):933-47.

Kang JI, Jeong DK, Choi $\mathrm{H}$. Effects of microcurrent and cryotherapy on C-reactive protein levels and muscle tone of patients with rotator cuff reconstruction. J Phys Ther Sci. 2018;30(1):37-41.

Kienbacher T, Habenicht R, Starek C, et al. The potential 
use of spectral electromyographic fatigue as a screening and outcome monitoring tool of sarcopenic back muscle alterations. J NeuroengRehabil. 2014; 11(1):106-17.

Kim D, Kim T, Jung D, et al. Effects of the Graston Technique and Self-myofascial Release on the Range of Motion of a Knee Joint. Journal of the Korean Society of Physical Medicine. 2014;9(4):455-63.

Kim S, Park H, Jung H. The Comparison of Effect of MC Intensity in Pain and ROM in Delayed Onset Muscle Soreness. Journal of the Korean Academy of Clinical Electrophysiology. 2009;7(1):1-6.

Kwon DR, Kim J, Kim Y, et al. Short-term microcurrent electrical neuromuscular stimulation to improve muscle function in the elderly: A randomized, double-blinded, sham-controlled clinical trial. Medicine (Baltimore). 2017;96(26):7407.

Lambert MI, Marcus P, Burgess T, et al. Electro-membrane microcurrent therapy reduces signs and symptoms of muscle damage. Med Sci Sports Exerc. 2002; 34(4):602-7.

Lee JH, Lee DK, Oh JS. The effect of Graston technique on the pain and range of motion in patients with chronic low back pain. J Phys Ther Sci. 2016;28(6): 1852-5.

Lee JW, Kang JS, Park SJ, et al. Effects of Inter-electrode Distance on Delayed Onset Muscle Soreness in Microcurrent Therapy. J Phys Ther Sci. 2013;25(11): 1451-4.

Lou JW, Bergquist AJ, Aldayel A, et al. Interleaved neuromuscular electrical stimulation reduces muscle fatigue. Muscle Nerve. 2017;55(2):179-89.

Lyons MF, Rouse ME, Baxendale RH. Fatigue and EMG changes in the masseter and temporalis muscles during sustained contractions. J Oral Rehabil. 1993;20(3): 321-31.

Millet GY, Lepers R. Alterations of neuromuscular function after prolonged running, cycling and skiing exercises.
Sports Med. 2004;34(2):105-16.

Moon JH, Jung JH, Won YS, et al. Immediate effects of Graston Technique on hamstring muscle extensibility and pain intensity in patients with nonspecific low back pain. J Phys Ther Sci. 2017;29(2):224-7.

Moraska A. Therapist education impacts the massage effect on postrace muscle recovery. Med Sci Sports Exerc. 2007;39(1):34-7.

Mori H, Ohsawa H, Tanaka TH, et al. Effect of massage on blood flow and muscle fatigue following isometric lumbar exercise. Med Sci Monit. 2004;10(5):173-8.

Nieman DC, Miller AR, Henson DA, et al. Effect of highversus moderate-intensity exercise on lymphocyte subpopulations and proliferative response. Int J Sports Med. 1994;15(4):199-206.

Resnick PB. Comparing the Effects of Rest and Massage on Return to Homeostasis Following Submaximal Aerobic Exercise: a Case Study. Int J Ther Massage Bodywork. 2016;9(1):4-10.

Senturk UK, Gunduz F, Kuru O, et al. Exercise-induced oxidative stress leads hemolysis in sedentary but not trained humans. J Appl Physiol (1985). 2005;99(4):1434-41.

Stanek J, Sullivan T, Davis S. Comparison of Compressive Myofascial Release and the Graston Technique for Improving Ankle-Dorsiflexion Range of Motion. J Athl Train. 2018.

Stulen FB, De Luca CJ. Muscle fatigue monitor: a noninvasive device for observing localized muscular fatigue. IEEE Trans Biomed Eng. 1982;29(12):760-8.

Tanaka TH, Leisman G, Mori H, et al. The effect of massage on localized lumbar muscle fatigue. BMC Complement Altern Med. 2002;2(1):1-9.

Todd I, Clothier RH, Huggins ML, et al. Electrical stimulation of transforming growth factor-beta 1 secretion by human dermal fibroblasts and the U937 human monocytic cell line. Altern Lab Anim. 2001;29(6): 693-701.

Whitcomb E, Monroe N, Hope-Higman J, et al. Demonstration 
of a microcurrent-generating wound care device for wound healing within a rehabilitation center patient population. J Am Coll Clin Wound Spec. 2012;4(2): $32-9$.
Yokoe A, Ohira M. Effect of cooling down after strenuous exercise on the quality of sleep the same day, and the level of fatigue the following morning. Sleep Medicine. 2017;40(1):e356. 